\title{
A New Approach to Dehydration of xylose to 2-furfuraldehyde Using a Mesoporous
}

\section{Niobium-based Catalyst}

José B. Gabriell-2. Victor Oliveiral. Talita Souza ${ }^{2}$. Izabela Padula ${ }^{2}$. Luiz C. A. Oliveira ${ }^{2}$. Leandro V. A. Gurgel ${ }^{1}$. Bruno E. L. Baêta ${ }^{1}$. Adilson C. Silva ${ }^{*}$

${ }^{1}$ Laboratory of Technological and Environmental Chemistry. Department of Chemistry. Institute of Exact and Biological Sciences (ICEB). Federal University of Ouro Preto. Campus Universitário Morro do Cruzeiro. Bauxita. 35400-000 Ouro Preto. Minas Gerais. Brazil

${ }^{2}$ Department of Chemistry. Institute of Exact Sciences (ICEX). Federal University of Minas Gerais (UFMG). Avenida Antônio Carlos. 6627. 31270-901 Belo Horizonte. Minas Gerais. Brazil.

*Corresponding author: A. C. da Silva; E-mail address: adilsonqui@ufop.edu.br/ adilsonufla@gmail.com; Phone number:+55 31 3559-1934. 
Tables: 04

Supplementary Table 1. Regression coefficients for the responses evaluated in the $2^{3}$ experimental design.

\begin{tabular}{|c|c|c|c|c|c|c|}
\hline $\mathbf{Y i}$ & $\begin{array}{c}\text { Regressn } \\
\text { Coeff. }\end{array}$ & $\begin{array}{l}\text { Std.Err. } \\
\text { Pure Err }\end{array}$ & $t(2)$ & value & $\begin{array}{c}-\mathbf{- 9 5 \%} \\
\text { Cnf.Limt }\end{array}$ & $\begin{array}{c}+95 \% \\
\text { Cnf.Limt }\end{array}$ \\
\hline Mean & -164.677 & 45.06818 & -3.65395 & 0.067413 & -358.590 & 29.23576 \\
\hline (1 & 813 & 0.50669 & 3.57756 & 0.07 & 367 & \\
\hline (2) $t$ & & & 1.60764 & 0.249 & 286 & 2792 \\
\hline (3) $C$ & & 0.13972 & 0.44714 & 0.69 & & 6363 \\
\hline$T\left({ }^{\circ} \mathrm{C}\right.$ & 4 & 0.00140 & -3.0 & 36 & & \\
\hline $2 \mathrm{~L}$ by $3 \mathrm{~L}$ & .001 & 0.00056 & -1.90745 & 0.196 & -0.003 & 0.00133 \\
\hline $1 \mathrm{~L}$ & .001 & 0.00073 & -1.0 & 0.4 & .004 & 0.00241 \\
\hline & .000 & 0.00056 & -0.8 & & .003 & 0.00192 \\
\hline & $\begin{array}{l}\text { Regressn } \\
\text { Coeff. }\end{array}$ & $\begin{array}{l}\text { Std.Err. } \\
\text { Pure Err }\end{array}$ & t ( & $p$-value & $\begin{array}{c}-\mathbf{9 5 \%} \\
\text { Cnf.Limt }\end{array}$ & Cnf.Limt \\
\hline $\mathbf{M}$ & -732.552 & 20.2 & -36 & 0.00 & -819.484 & -645.620 \\
\hline (1) 1 & 7.776 & 0.22715 & 34.2 & 0.0 & 6.798 & 8.753 \\
\hline (3) & 1 & 0.0 & 18.7 & 0.0 & & 446 \\
\hline (2) $t$ & 53 & 0.0 & 11.8 & 0.0 & 58 & .768 \\
\hline$T\left({ }^{\circ} \mathrm{C}\right.$ & -0.018 & 0.00063 & -29.1 & 0.0 & -0.021 & 0.016 \\
\hline $1 \mathrm{~L} \mathrm{~b}$ & -0.006 & 0.00033 & -17.0 & 0.00 & -0.007 & -0.004 \\
\hline & -0.002 & 0.00025 & -9.6939 & 0.01 & -0.003 & -0.001 \\
\hline $2 \mathrm{~L} \mathrm{~b}$ & 0.000 & 0.00025 & 0.5492 & 0.638018 & -0.001 & 0.001 \\
\hline $\mathbf{S}$ & $\begin{array}{c}\text { Regressn } \\
\text { Coeff. }\end{array}$ & $\begin{array}{l}\text { Std.Err. } \\
\text { Pure Err }\end{array}$ & $t(2)$ & $\begin{array}{c}p- \\
\text { value }\end{array}$ & $\begin{array}{c}-95 \% \\
\text { Cnf.Limt }\end{array}$ & $\begin{array}{r}+9 \\
\text { Cnf. }\end{array}$ \\
\hline & 306.5686 & 46.98692 & 6.5246 & 0.022694 & 104.4002 & 508.7370 \\
\hline (1) $T$ & -2.3608 & 0.52826 & -4.4690 & 0.046598 & -4.6338 & -0.0879 \\
\hline (3) $C X R(\%)(\mathrm{L}$ & -1.7158 & 0.14567 & -11.7790 & 0.007130 & -2.3426 & -1.0891 \\
\hline $1 \mathrm{~L}$ by $3 \mathrm{~L}$ & 0.0074 & 0.00076 & 9.7094 & 0.010442 & 0.0041 & 0.0107 \\
\hline$T\left({ }^{\circ} \mathrm{C}\right)(\mathrm{Q})$ & 0.0051 & 0.00146 & 3.4756 & 0.073742 & -0.0012 & 0.0113 \\
\hline & & & -0.0334 & 0.976409 & -0.4803 & 0.4729 \\
\hline $2 \mathrm{~L}$ by $3 \mathrm{~L}$ & -0.0012 & 0.00058 & -2.0215 & 0.180603 & -0.0037 & 0.0013 \\
\hline $1 \mathrm{~L}$ by $2 \mathrm{~L}$ & 0.0003 & 0.00058 & 0.5041 & 0.664239 & -0.0022 & 0.0028 \\
\hline
\end{tabular}


Supplementary Table 2. ANOVA results for response yield (\%) evaluated in the $2^{3}$ experimental design.

\begin{tabular}{|c|c|c|c|c|c|}
\hline \multirow[t]{2}{*}{ Factor } & \multicolumn{5}{|c|}{$\begin{array}{c}\text { ANOVA; Var: Yield (\%); } R^{2}=0.93033 ; R^{2 \mathrm{a}} \mathrm{d} ; 0.76777 \text { (Spreadsheet5) } 3 \text { factors. } 1 \text { Blocks. } 11 \\
\text { Runs; MS Pure Error }=10.9099 \text { DV: Yield (\%) }\end{array}$} \\
\hline & SS & $\mathrm{df}$ & MS & $F$ & $p$-value \\
\hline $\begin{array}{c}(1) T \\
\left({ }^{\circ} \mathrm{C}\right)(\mathrm{L})\end{array}$ & 662.116 & 1 & 662.1161 & 60.68947 & 0.016081 \\
\hline$T\left({ }^{\circ} \mathrm{C}\right)(\mathrm{Q})$ & 99.188 & 1 & 99.1883 & 9.09159 & 0.094636 \\
\hline $\begin{array}{c}(2) t \\
(\min )(\mathrm{L})\end{array}$ & 24.500 & 1 & 24.5000 & 2.24567 & 0.272723 \\
\hline $\begin{array}{l}(3) C X R \\
(\%)(\mathrm{L})\end{array}$ & 253.575 & 1 & 253.5752 & 23.24267 & 0.040433 \\
\hline $1 \mathrm{~L}$ by $2 \mathrm{~L}$ & 7.762 & 1 & 7.7618 & 0.71145 & 0.487764 \\
\hline $1 \mathrm{~L}$ by $3 \mathrm{~L}$ & 10.951 & 1 & 10.9512 & 1.00379 & 0.421923 \\
\hline $2 \mathrm{~L}$ by $3 \mathrm{~L}$ & 39.694 & 1 & 39.6941 & 3.63835 & 0.196703 \\
\hline$\frac{\text { *Lack of }}{\underline{\text { Fit }}}$ & 60.390 & 1 & 60.3900 & 5.53534 & $* 0.142921$ \\
\hline Pure Error & 21.820 & 2 & 10.9099 & & \\
\hline Total SS & 1179.996 & 10 & & & \\
\hline
\end{tabular}

*When $p$-value $>0.05$, lack of fit of mathematical model is no significant. 
Supplementary Table 3. ANOVA results for response conversion (\%) evaluated in the experimental design.

\begin{tabular}{|c|c|c|c|c|c|}
\hline \multirow{2}{*}{ Factor } & \multicolumn{5}{|c|}{$\begin{array}{c}\text { ANOVA; Var.:Conversion (\%); } R^{2}=0.99627 ; R^{2} \text { adj:0.98756 (Spreadsheet5) } 3 \text { factors. } 1 \text { Blocks. } \\
\text { 11 Runs; MS Pure Error=2.192633 DV: Conversion (\%) }\end{array}$} \\
\hline & SS & $\mathrm{df}$ & MS & $F$ & $p$-value \\
\hline $\begin{array}{c}(1) T \\
\left({ }^{\circ} \mathrm{C}\right)(\mathrm{L})\end{array}$ & 7287.87 & 1 & 7287.866 & 3323.796 & 0.000301 \\
\hline $\begin{array}{c}T \\
\left({ }^{\circ} \mathrm{C}\right)(\mathrm{Q})\end{array}$ & 1865.19 & 1 & 1865.193 & 850.663 & 0.001173 \\
\hline $\begin{array}{c}(2) t \\
(\min )(\mathrm{L})\end{array}$ & 401.86 & 1 & 401.861 & 183.278 & 0.005412 \\
\hline $\begin{array}{l}(3) C X R \\
(\%)(\mathrm{L}) \\
\end{array}$ & 417.60 & 1 & 417.605 & 190.458 & 0.005210 \\
\hline $1 \mathrm{~L}$ by $2 \mathrm{~L}$ & 206.05 & 1 & 206.045 & 93.971 & 0.010475 \\
\hline $1 \mathrm{~L}$ by $3 \mathrm{~L}$ & 639.03 & 1 & 639.031 & 291.445 & 0.003414 \\
\hline $2 \mathrm{~L}$ by $3 \mathrm{~L}$ & 0.66 & 1 & 0.661 & 0.302 & 0.638018 \\
\hline *Lack of & 36.13 & 1 & 36.125 & 16.476 & $* 0.055675$ \\
\hline $\begin{array}{l}\text { Pure } \\
\text { Error }\end{array}$ & 4.39 & 2 & 2.193 & & \\
\hline Total SS & 10858.77 & 10 & & & \\
\hline
\end{tabular}

*When $p$-value $>0.05$, lack of fit of mathematical model is no significant. 
Supplementary Table 4. ANOVA results for response selectivity (\%) evaluated in the $2^{3}$ experimental design.

\begin{tabular}{|c|c|c|c|c|c|}
\hline \multirow[b]{2}{*}{ Factor } & \multicolumn{5}{|c|}{$\begin{array}{l}\text { ANOVA; Var.:Selectivity (\%); } R^{2}=0.97024 ; R^{2} \text { adj:0.90079 (Spreadsheet5) } 3 \text { factors. } 1 \text { Blocks. } \\
11 \text { Runs; MS Pure Error=11.85863 DV: Selectivity (\%) }\end{array}$} \\
\hline & SS & $\mathrm{df}$ & MS & $F$ & $p$-value \\
\hline $\begin{array}{l}(1) T \\
\left({ }^{\circ} \mathrm{C}\right)(\mathrm{L})\end{array}$ & 282.388 & 1 & 282.388 & 23.8128 & 0.039521 \\
\hline $\begin{array}{l}T \\
\left({ }^{\circ} \mathrm{C}\right)(\mathrm{Q})\end{array}$ & 143.252 & 1 & 143.252 & 12.0800 & 0.073742 \\
\hline $\begin{array}{l}(2) t \\
(\min )(\mathrm{L})\end{array}$ & 2.071 & 1 & 2.071 & 0.1746 & 0.716638 \\
\hline $\begin{array}{l}(3) C R X \\
(\%)(\mathrm{L})\end{array}$ & 2768.052 & 1 & 2768.052 & 233.4208 & 0.004257 \\
\hline $1 \mathrm{~L}$ by $2 \mathrm{~L}$ & 3.014 & 1 & 3.014 & 0.2541 & 0.664239 \\
\hline $1 \mathrm{~L}$ by $3 \mathrm{~L}$ & 1117.936 & 1 & 1117.936 & 94.2719 & 0.010442 \\
\hline $2 \mathrm{~L}$ by $3 \mathrm{~L}$ & 48.462 & 1 & 48.462 & 4.0866 & 0.180603 \\
\hline Fit & 110.187 & 1 & 110.187 & 9.2917 & *0.092873 \\
\hline $\begin{array}{l}\text { Pure } \\
\text { Error }\end{array}$ & 23.717 & 2 & 11.859 & & \\
\hline Total SS & 4499.078 & 10 & & & \\
\hline
\end{tabular}

*When $p$-value $>0.05$, lack of fit of mathematical model is no significant. 


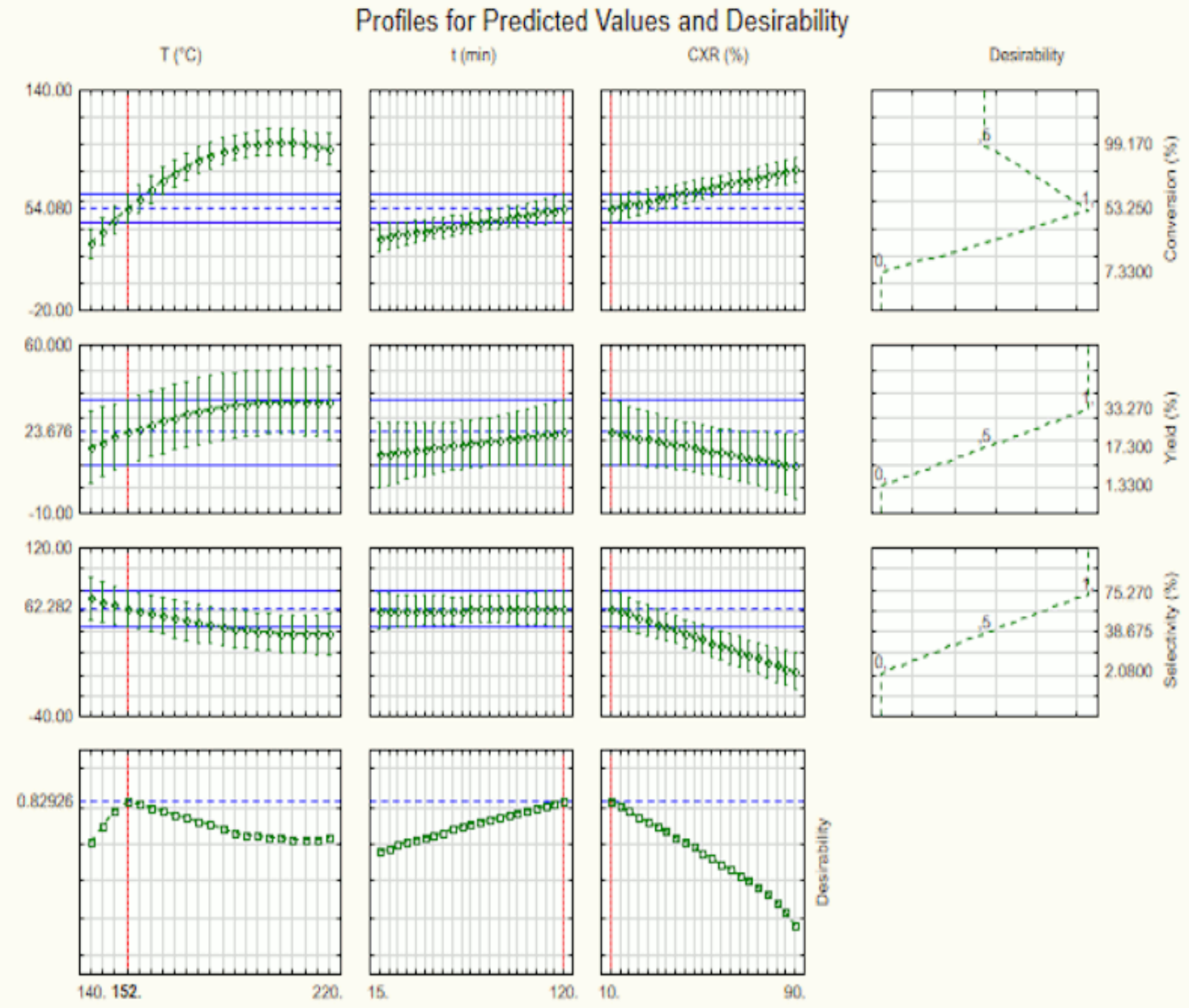

Supplementary Figure 1. Predicted values by the desirability tool for all responses studied. 
